\title{
Quantum Spectroscopy of Plasmonic Nanostructures
}

\author{
Dmitry A. Kalashnikov, Zhenying Pan, Arseniy I. Kuznetsov, and Leonid A. Krivitsky* \\ Data Storage Institute, Agency for Science Technology and Research (A-STAR), \\ 5 Engineering Drive I, 117608, Singapore
}

(Received 11 December 2013; revised manuscript received 6 February 2014; published 25 March 2014)

We use frequency-entangled photons, generated via spontaneous parametric down conversion, to measure the broadband spectral response of an array of gold nanoparticles exhibiting Fano-type plasmon resonance. Refractive-index sensing of a liquid is performed by measuring the shift of the array resonance. This method is robust in excessively noisy conditions compared with conventional broadband transmission spectroscopy. Detection of a refractive-index change is demonstrated with a noise level 70 times higher than the signal, which is shown to be inaccessible with the conventional transmission spectroscopy. Use of low-photon fluxes makes this method suitable for measurements of photosensitive biosamples and chemical substances.

DOI: 10.1103/PhysRevX.4.011049

Subject Areas: Nanophysics, Optics, Quantum Physics

\section{INTRODUCTION}

New insights into understanding the behavior of materials at the nanoscale and progress in nanofabrication capabilities have triggered great interest in the study of plasmonic nanostructures. One practical application of these structures is the development of sensing technology. Plasmonic sensors benefit from their biocompatibility and high sensitivity [1-4]. With the sensitivity of the sensors approaching the atto-Molar scale, as well as sensing of photosensitive substances, it is important that the analyte is not damaged or modified by the probing light. Thus, we need to use extremely small doses of light, possibly down to the level of single photons, and highly sensitive equipment for the readout of the sensor response. For sensing with low-photon counts, the main practical issue is careful suppression of the influence of the background noise. This is challenging with conventional spectroscopy, but quantum optics can be exploited to effectively address this issue [5-7].

Interfacing quantum optics with plasmonics is an area of active research. Plasmonic nanostructures are used as efficient optical antennas for increasing the collection efficiency of light, emitted by solid-state single-photon sources [8-16]. They are also shown to preserve quantum entanglement [17-19] and squeezing [20,21], thus making it possible to use them in subwavelength quantum optical networks [22].

\footnotetext{
*Corresponding author. Leonid_Krivitskiy@dsi.a-star.edu.sg

Published by the American Physical Society under the terms of the Creative Commons Attribution 3.0 License. Further distribution of this work must maintain attribution to the author(s) and the published article's title, journal citation, and DOI.
}

In this work, we demonstrate advantages of using frequency-entangled photons, generated via spontaneous parametric down conversion (SPDC), in revealing the spectroscopic response of a plasmonic refractive-index sensor under noisy conditions. The technique operates at a single-photon level and is more robust to environmental noise, compared to conventional transmission spectroscopy.

\section{QUANTUM SPECTROSCOPY}

In the SPDC, a pump photon traveling in a medium with quadratic susceptibility occasionally splits into a pair of photons, referred to as the signal and idler [23,24]. Signal and idler photons are created almost simultaneously (within tens of $f s$ ) with their energies and momenta obeying the conservation laws:

$$
\omega_{p}=\omega_{s}+\omega_{i} \quad \vec{k}_{p}=\vec{k}_{s}+\vec{k}_{i},
$$

where $\omega_{p, s, i}$ and $\vec{k}_{p, s, i}$ are the energies and the wave vectors of the pump, signal, and idler photons, respectively. Signal and idler photons are detected with two single-photon avalanche photodiodes (APD). Their pairwise correlation is measured by an electronic coincidence circuit, which produces a signal (coincidence) if APD counts arrive within the fixed time window, typically of about several $n s$. Dedicated techniques allow one to obtain the widths of the SPDC spectra in a given spatial mode in the range of several hundreds of nanometers [25-28].

The sample under investigation is placed in the path of one of the photons (idler), and the frequency selection is performed over another photon (signal) by a monochromator [29-33] [see Fig. 1(a)]. Since the sum of energies of the down-converted photons is equal to the energy of the pump, spectral selection over the signal photon defines the frequency of the correlated idler photon. Following 


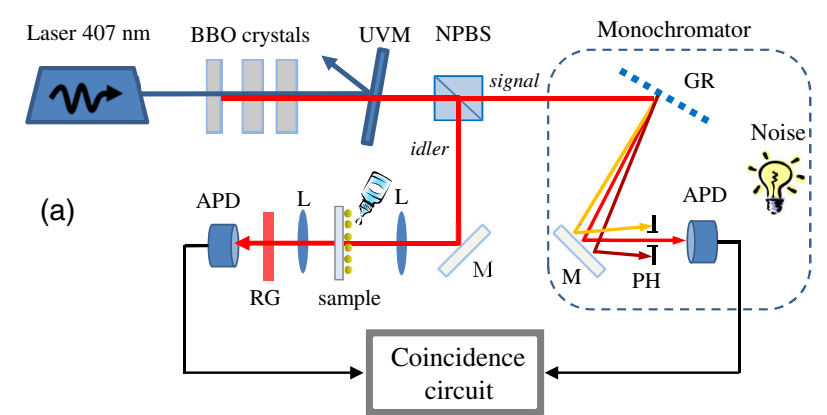

(b)

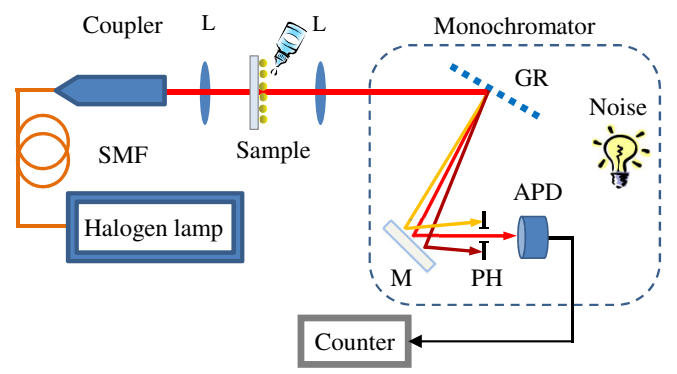

FIG. 1. (a) Quantum spectroscopy setup. Diode laser at $407 \mathrm{~nm}$ is used as a pump for a set of three BBO crystals cut for type-I SPDC; the UV mirror (UVM) reflects the pump beam and transmits the SPDC signal; a nonpolarizing beam splitter (NPBS) splits the SPDC signal into two arms; in one arm, there is a monochromator, which consists of a mirror (M), a diffraction grating (GR), a pinhole, and an APD. In another arm, there is a sample, which represents an array of gold nanoparticles assembled into a cell. The SPDC signal is focused onto the sample by achromatic lenses (L), filtered by a red glass filter (RG), and detected by the APD. Counts of the two APDs are sent to a coincidence circuit; the source of the noise is provided by the external APD, illuminated by the lamp (not shown), whose signal is mixed electronically with the signal of the APD in the signal channel. (b) Transmission spectroscopy setup. A halogen lamp is used as a source of the broadband light; the light after a single mode fiber (SMF) is collimated by a coupler, focused by lenses onto the sample, filtered by the monochromator, and detected by the APD.

Ref. [29], the number of coincidences between two APDs is proportional to the spectral function of the sample $T(\omega)$ at the frequency of the idler photon,

$$
R\left(\omega_{s}\right) \sim T\left(\omega_{p}-\omega_{s}\right)
$$

In contrast to the conventional transmission spectroscopy, the spectral selection is performed in the channel without the structure under test. Following the analogy with imaging experiments, we refer to the method as quantum (ghost) spectroscopy.

The remarkable feature of the quantum spectroscopy is its robustness against the environmental noise, which allows one to conduct measurements at extremely lowphoton fluxes. The noise typically includes background optical noise and electronic noise of the APDs. Let us denote the number of SPDC photocounts as $S_{s, i}=\eta_{s, i} P$, where $P$ is the number of photon pairs, produced by the
SPDC source, and $\eta_{s, i}$ is the quantum efficiency of the signal and idler channel, respectively. The number of coincidences is proportional to the probability of joint detection by the APDs in two channels:

$$
R\left(\omega_{s}\right)=\eta_{s} \eta_{i} P .
$$

Assuming that noise photocounts of the two APDs are uncorrelated, the number of noise coincidences $R_{N}$ is given by accidental overlap of photocounts within the time window of the coincidence circuit $\Delta t$. It also includes components due to accidental overlap of SPDC and noise photocounts:

$$
R_{N}=N_{s} N_{i} \Delta t+S_{s} N_{i} \Delta t+N_{s} S_{i} \Delta t,
$$

where $N_{s}$ and $N_{i}$ are the number of noise photocounts of APDs in signal and idler channels, respectively. The total number of coincidences is given by

$$
R_{\text {Total }}=R\left(\omega_{s}\right)+R_{N} .
$$

Even though $N_{s}, N_{i} \gg S_{s}, S_{i}$, the number of noise coincidences $R_{N}$ is strongly suppressed compared to $R\left(\omega_{s}\right)$ because of a narrow coincidence time window $\Delta t$. Thus, the spectral response of the structure can be revealed from $R_{\text {Total }}$ even under excessive noise situations.

\section{COMPARISON TO THE CONVENTIONAL TRANSMISSION SPECTROSCOPY}

In conventional transmission spectroscopy, the sample is placed between the light source and the monochromator; the transmission spectrum is obtained directly from APD photocounts [see Fig. 1(b)]. The signal-to-noise ratio (SNR) for the transmission spectroscopy is given by $\mathrm{SNR}_{T}=\eta_{s} P / N_{s}$. For the quantum spectroscopy, the $\mathrm{SNR}$ is given by $\mathrm{SNR}_{Q}=R\left(\omega_{s}\right) / R_{N}=\eta_{i} \eta_{s} P / N_{s} N_{i} \Delta t$, see Eqs. (3) and (4), where it is assumed that $N_{s} N_{i} \gg S_{s} N_{i} ; N_{s} S_{i}$. The ratio of the two SNRs is given by

$$
\mathrm{SNR}_{Q} / \mathrm{SNR}_{T}=\eta_{i} / N_{i} \Delta t .
$$

The advantage in the SNR for the quantum spectroscopy is provided by the use of high-quantum efficiency, lownoise APD in the idler channel, and the narrow coincidence time window $\Delta t$.

\section{THE PLASMONIC ARRAY SENSOR}

We use an array of metal nanoparticles which has a narrow Fano-type plasmon resonance, due to diffractive coupling of localized surface plasmons [34-39]. The array acts as a metallic grating, and when the wavelength of incident light gets close to the Rayleigh cutoff wavelength, the diffracted wave propagates along the surface [40]. This effect is referred to as Wood-Rayleigh's anomaly. 
If the anomaly wavelength is close to the plasmon resonance of individual nanoparticles, then the collective plasmonic mode is excited. Excitation of the mode results in a narrow Fano-type resonance in the transmission spectrum of the array. The position and shape of the Fano resonance depend on the shape of the nanoparticles, distance between them, and refractive index of the surrounding medium. High sensitivity to the local change of the refractive index, as well as the high-quality $(\mathrm{Q})$ factor of the nanoparticle array resonance, constitutes the basis for its sensing applications $[37,41]$.

The nanoparticle array is fabricated using a combined method of nanosphere lithography with femtosecond laserinduced transfer, which allows production of large-scale periodic arrays of spherical nanoparticles [37]. A hexagonal array with dimensions of approximately $1 \times 1 \mathrm{~mm}^{2}$ is fabricated with a particle diameter of $130 \mathrm{~nm}$ and a hexagonal lattice period of $1.1 \mu \mathrm{m}$ [see Figs. 2(a) and 2(b)]. The fabricated particles are partially embedded (around twothirds) into a polydimethylsiloxane (PDMS) substrate, with a part (around one-third) above the substrate surface (this is accessible to the local environment). With the sample covered by a second PDMS layer, the nanoparticles are in a homogeneous surrounding and the array provides a Fano resonance centered at $806 \mathrm{~nm}$ with a quality factor $\mathrm{Q} \approx 27$, where $Q$ is defined as the ratio of the resonance wavelength to the resonance width, defined from the fit using the Fano formula (see details below).

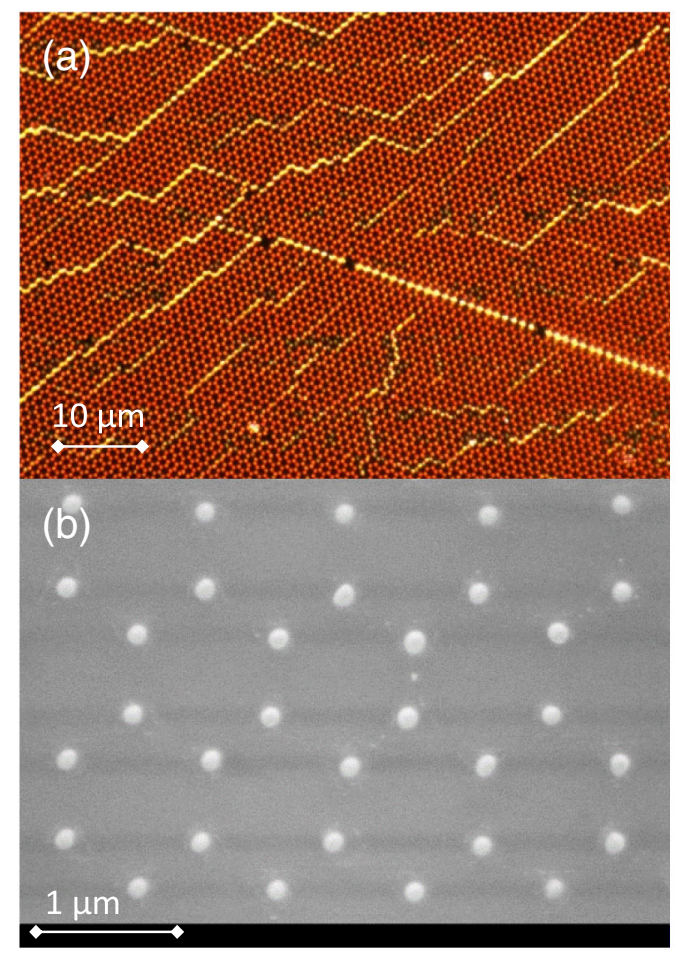

FIG. 2. Images of the plasmonic nanoparticle array on a PDMS substrate. (a) Dark-field microscope image at $10 \times$ magnification. (b) Scanning electron microscope (SEM) image.
The refractive-index sensitivity is measured by removing the cover PDMS layer and adding testing glycerin-water solutions with different concentrations on top of the array. The sensitivity is calculated as a ratio of the resonance shift in $\mathrm{nm}$ to the change of the refractive index of the testing solution.

\section{EXPERIMENTAL SETUP}

The quantum spectroscopy setup is shown in Fig. 1(a). Three bulk BBO crystals (Dayoptics) with thicknesses of $0.3 \mathrm{~mm}, 0.5 \mathrm{~mm}$, and $0.5 \mathrm{~mm}$ are pumped by a continuouswave vertically polarized diode laser at $407 \mathrm{~nm}$ (Omicron PhoxX-405-60). The BBOs are cut for type-I collinear SPDC. One of the BBOs is set for the frequency-degenerate regime, while two others are detuned from the degenerate regime by tilting their optical axis [26]. The resulting SPDC light source has a spectral width of around $250 \mathrm{~nm}$ and is shown in Fig. 3. The pump is eliminated by a UV mirror, and the SPDC is split by a nonpolarizing beam splitter (NPBS). In one arm of the NPBS, we inserted a home-built diffraction grating monochromator (Thorlabs GR-1205) with resolution $1.5 \mathrm{~nm}$. The light after the monochromator is detected by an avalanche photodiode (APD, Perkin Elmer SPCM-AQRH-14FC).

The sample is attached to a glass substrate and mounted in another arm of the NPBS in the focal plane of two confocal achromatic lenses with focus length $f=60 \mathrm{~mm}$. A mechanical translation stage (not shown) is used to position the sample. The focused light has a diameter of $300 \mu \mathrm{m}$ on the sample surface. The sample area with the array is surrounded by PDMS walls and covered by a thin glass plate forming a cell. The refractive index of the medium surrounding the array can be changed by adding different solvents inside the cell. The SPDC light passes through the broadband red glass filter (RG), and then it is coupled into the multimode fiber and detected by the APD

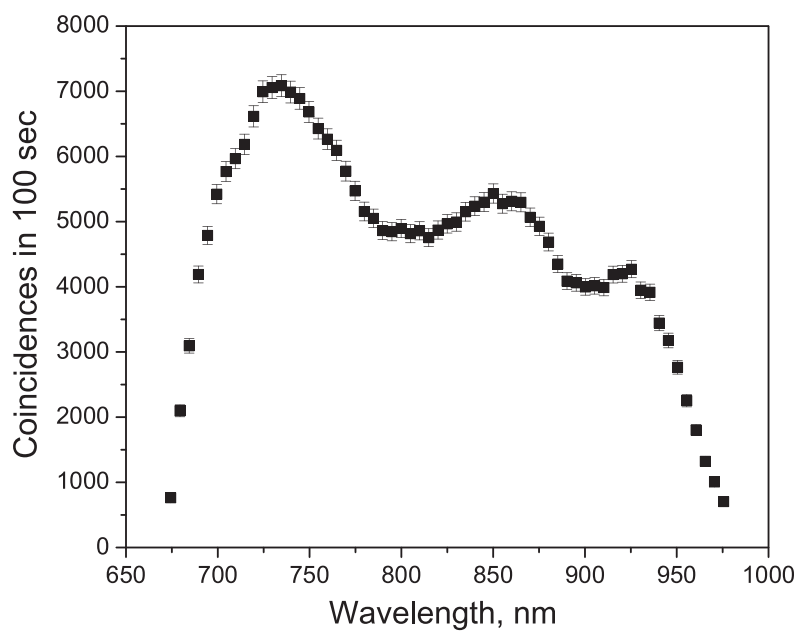

FIG. 3. Spectrum of coincidences of SPDC from three BBO crystals. The error bars are the standard deviations. 
(Perkin Elmer SPCM-AQRH-14FC). The signals from the two APDs are sent to the coincidence circuit with the time window of $\Delta t=5 \mathrm{~ns}$ (Ortec $567 \mathrm{TAC} / \mathrm{SCA})$. The number of coincidences is measured as a function of wavelength, selected by the monochromator. The step size of the wavelength selection is $3 \mathrm{~nm}$.

Noisy conditions are emulated by artificially adding photocounts of an additional APD (not shown), illuminated by a lamp, to the signal of the APD in the monochromator. This approach allows one to exclude the spectral dependence of the noise. The level of noise is controlled by changing the driving current of the lamp.

Results of the quantum spectroscopy are compared with those obtained by the transmission spectroscopy [see Fig. 1(b) for the experimental setup]. The light from the lamp after the single mode fiber (SMF) is sent through the sample under investigation, using the same optical system as described above, and then coupled to the monochromator. APD photocounts are recorded versus the wavelength, selected by the monochromator.

The experimental procedure in both cases includes transmission measurements of the sample substrate and the array. The resulting transmission spectrum is calculated as the ratio of the array and the substrate spectra.

\section{RESULTS AND DISCUSSION}

To ensure that the SPDC bandwidth fully covers the range of the structure's Fano resonance, we measure the sample transmission with the quantum spectroscopy setup. The nanoparticle array is covered by a layer of PDMS to create homogeneous surroundings. The refractive index of the PDMS is about 1.4 [42]. The obtained spectrum is shown in Fig. 4. It reveals the Fano-type resonance with the distinctive asymmetrical shape. The gray solid curve in Fig. 4 shows the fitting of the experimental results with the Fano formula [38,39], with the following parameters: resonance wavelength $\lambda_{R}=806 \mathrm{~nm}$, resonance width $\Delta \lambda=30 \mathrm{~nm}$, and Fano (asymmetry) parameter $\mathrm{F}=-16$. This gives the resonance quality factor $\mathrm{Q}=\lambda_{R} / \Delta \lambda \approx 27$.

Refractive-index sensing is demonstrated by depositing a few drops (around $10 \mu \mathrm{l}$ ) of the testing solution of glycerin in water on the sample surface. In two different measurements, the glycerin concentrations are $40 \%$ and $50 \%$, with the corresponding refractive indexes of the solutions of 1.384 and 1.398 , respectively (the refractive-index values are taken from Ref. [43] for $20^{\circ} \mathrm{C}$ ). The refractive index of the solution of $50 \%$ glycerin in water is close to the refractive index of PDMS. Between subsequent measurements, the sample surface is washed with methanol and dried to remove the previous testing solution. Transmission spectra obtained at different glycerin concentrations using the conventional transmission spectroscopy are shown in Fig. 5(a). They demonstrate the redshift of the Fano resonance minima of about $8 \mathrm{~nm}$ when the glycerin concentration changes from $40 \%$ to $50 \%$. This corresponds

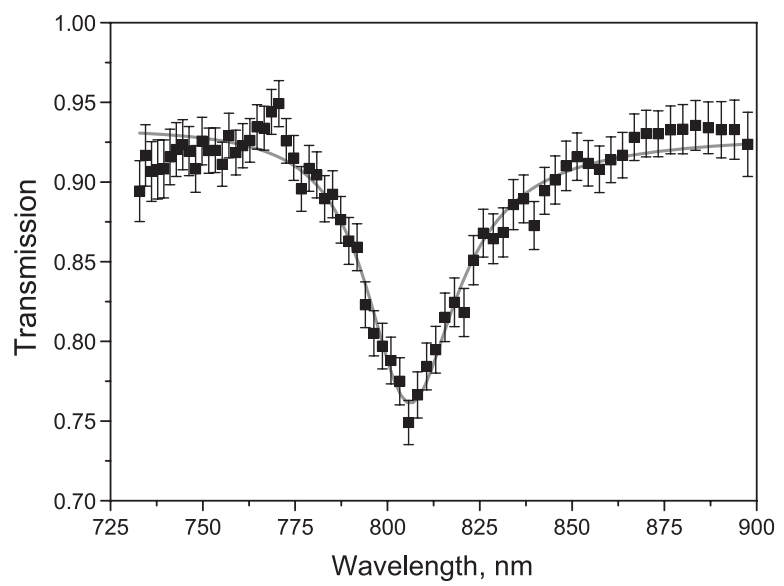

FIG. 4. Transmission spectrum of the nanoparticle array in homogeneous PDMS surroundings measured via quantum spectroscopy. The gray solid line is a fit with the Fano formula $\left(\mathrm{R}^{2}=0.94\right)$. The error bars are the standard deviations.

to the sensitivity value of $570 \mathrm{~nm} / \mathrm{RIU}$, which is a characteristic value for refractive-index sensing with plasmonic nanoparticles. Measurements performed by the quantum spectroscopy show similar results [see Fig. 5(b)].

It is important to emphasize that for the comparison of the two methods, all the measurements are performed with the same average flux of photons in the channel with the monochromator (in the vicinity of the resonance) equal to $10^{3}$ photocounts/sec. The measurements are also performed for the same acquisition time: Each point of the spectrum is obtained from the average of 20 measurements, each acquired for $20 \mathrm{~s}$.

Results of the measurements performed under noisy conditions for the case of the conventional transmission spectroscopy are shown in Figs. 5(a), 5(c), and 5(e). The noise levels are equal to $10^{2}$ photocounts/s in Fig. 5(a), $2 \times 10^{4}$ photocounts/s in Fig. 5(c), and $7 \times 10^{4}$ photocounts $/ \mathrm{s}$ in Fig. 5(e). With an increasing noise level, the resonance profile gets shallower and less pronounced. The maximum amount of noise at which it is feasible to perform sensing of the refractive-index change is given by the interplay of the spectral function of the sample, the sensitivity of the sensor, the average photon flux, the acquisition time, and the efficiency of the detection system [5]. From the experimental data in Fig. 5(e), one can see that when the noise is about 70 times larger than the signal, the resonance positions for both glycerin concentrations completely overlap, making it impossible to observe any refractive-index change.

The results obtained via the quantum spectroscopy are shown in Figs. 5(b), 5(d), and 5(f). The noise levels are equal to $10^{3}$ photocounts/s in Fig. $5(\mathrm{~b}), 2 \times 10^{4}$ photocounts/s in Fig. 5(d), and $7 \times 10^{4}$ photocounts $/ \mathrm{s}$ in Fig. 5(f). In contrast to the transmission spectroscopy, the shape and the depth of the resonance do not significantly change. The resonance position remains distinguishable, even when the noise is 

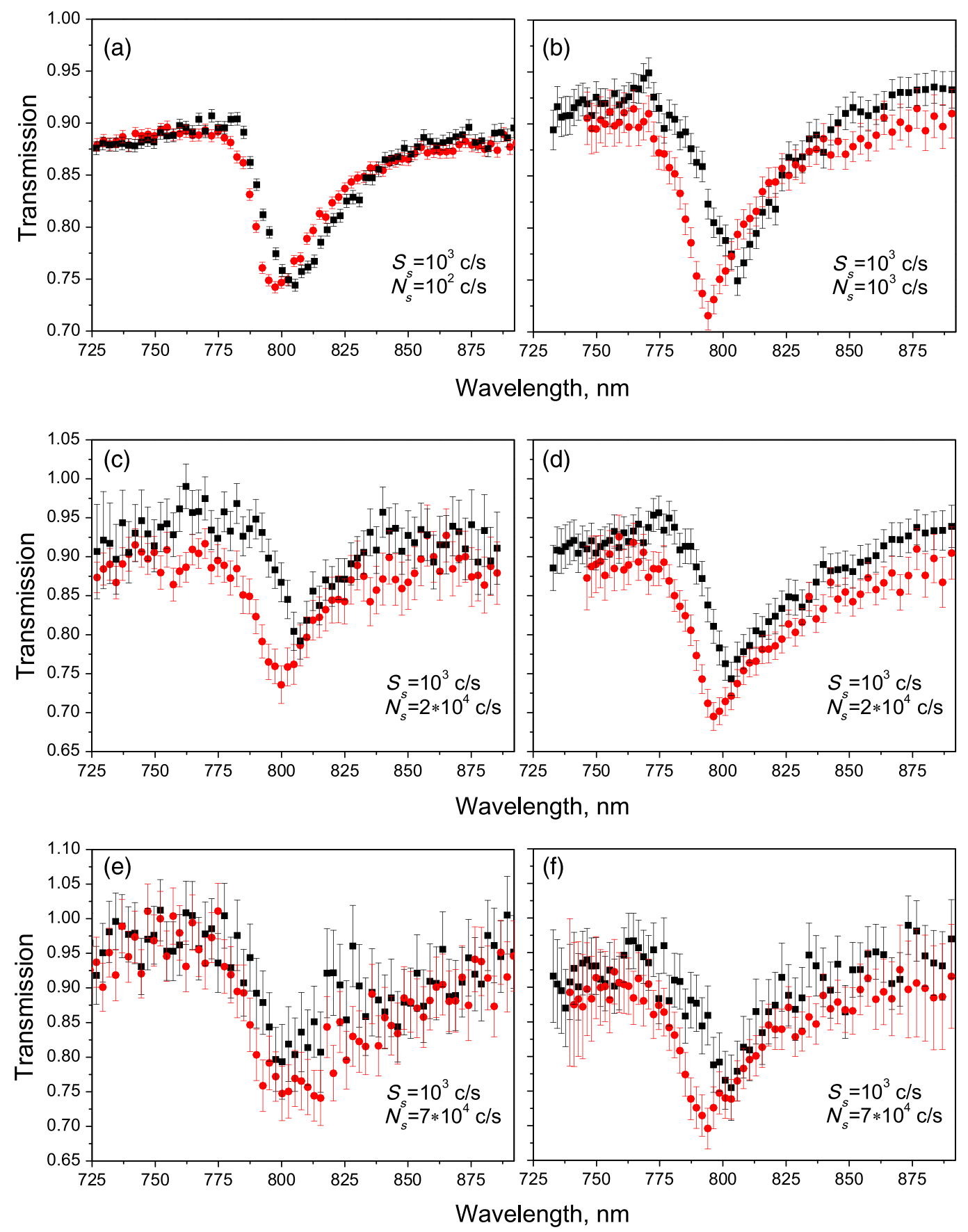

FIG. 5. Transmission spectrum of the nanoparticle array with different concentrations of glycerin-water solution on top, measured at different noise conditions. Red circles correspond to $40 \%$ glycerin concentration and black squares are assigned to $50 \%$ glycerin concentration. Results in (a), (c), and (e) are obtained by the conventional transmission spectroscopy with different noise levels of $10^{2}$ photocounts $/ \mathrm{s}, 2 \times 10^{4}$ photocounts/s, and $7 \times 10^{4}$ photocounts/s, respectively. Results in (b), (d), and (f) are obtained by the quantum spectroscopy with different noise levels: $10^{3}$ photocounts $/ \mathrm{s}, 2 \times 10^{4}$ photocounts $/ \mathrm{s}$, and $7 \times 10^{4}$ photocounts $/ \mathrm{s}$, respectively. The signal in the channel with the monochromator is the same for all the experiments in (a)-(f) and is equal to $10^{3}$ photocounts/s. The error bars are the standard deviations.

70 times larger than the signal in the monochromator [see Fig. 5(f)]. The only effect of the noise is a slight decrease in the accuracy of the resonance measurements due to the contribution of noise coincidences. However, the difference between the resonance minima remains the same as in the case of low noise. This shows that quantum spectroscopy has high resistance to noise and can be used with extremely low-photon fluxes, which are not possible 
with conventional transmission spectroscopy. This makes it very promising for sensing of photosensitive biological substances. Note that slight discrepancies in the shapes of Fano resonances in Figs. 5(a) and 5(b) are most likely caused by experimental uncertainties in setting the angle of incidence of the sensing light on the sample.

Let us estimate the improvement in the SNR by using the quantum spectroscopy compared to the transmission spectroscopy from Eq. (6). In our experiment, the width of the coincidence time window is set to $\Delta t=5 \mathrm{~ns}$. It is limited by the jitter of the APD (around $400 \mathrm{ps)} \mathrm{and} \mathrm{the}$ finite time resolution of the coincidence circuit (around $3 \mathrm{~ns}$ ). The noise of the APD in the idler channel is $N_{i}=10^{5}$ photocounts/s. It is contributed by an optical noise and photocounts from the detection of broadband idler photons at wavelengths that are not correlated with the wavelength of a signal photon, selected by the monochromator. Finally, the quantum efficiency in the idler channel is approximately $\eta_{i}=5 \%$. It accounts for the quantum efficiency of the APD $\left(\eta_{\mathrm{APD}}=50 \%\right)$ and optical losses in the idler channel. From Eq. (6), we estimate the resulting improvement in the SNR using quantum spectroscopy $\mathrm{SNR}_{Q} / \mathrm{SNR}_{T} \approx 100$.

For experiments with actual light-sensitive structures, it would be advantageous to place the sample just after the monochromator in the signal beam. In this case, the sample will be illuminated by a spectrally filtered light with fewer photons, compared to the present configuration. However, since the total number of detected photon pairs will remain the same, there will be no improvement in the resulting SNR.

The ratio $\mathrm{SNR}_{Q} / \mathrm{SNR}_{T}$ can be significantly increased by using an additional monochromator in the idler arm. The two monochromators should select exactly conjugated frequencies of signal and idler photons, given by Eq. (1). It would be feasible to decrease $N_{i}$ by at least 2 orders of magnitude. Furthermore, by using commercially available low-jitter APDs (around $50 \mathrm{ps}$ ) and time-to-digital converters with timing resolution on the order of around $10 \mathrm{ps}$ [44], it would be possible to set the coincidence time window to about $100 \mathrm{ps}$. Given this, it is feasible to reach a ratio of $\mathrm{SNR}_{Q} / \mathrm{SNR}_{T}$ as high as $5 \times 10^{5}$.

\section{CONCLUSION}

In conclusion, a new method for refractive-index sensing at extremely low-photon fluxes and under excessive noisy conditions is demonstrated. The method combines the use of entangled photon pairs, generated via spontaneous parametric down conversion, and plasmonic nanoparticle arrays with Fano resonance. Pairwise correlation between the photons allows spectral measurements with cancellation of uncorrelated background optical noise, and electronic noise of the detectors.

Using this method, detection of a refractive-index change of 0.014 is demonstrated with a noise level 70 times higher than the signal. The measured resonance shift of $8 \mathrm{~nm}$ corresponds to the relatively high refractive-index sensitivity of around $570 \mathrm{~nm} / \mathrm{RIU}$ provided by the Fano resonance of the nanoparticle array. It is shown that conventional spectroscopy fails to provide any sensing results in such excessive noisy conditions.

The developed approach will contribute to further progress in the application of plasmonic sensors. In particular, it will allow nondisturbing measurements of ultrasmall concentrations of chemicals (at atto-Molar scale), and sensing of photosensitive substances, which can be affected by the probing light.

\section{ACKNOWLEDGMENTS}

We acknowledge Manuel R. Gonçalves and Othmar Marti from the Institute of Experimental Physics, Ulm University for preparation of nanoparticle arrays on glass substrates by nanosphere lithography. We acknowledge Carl Zeiss Pte Ltd (Singapore) for assistance with the SEM imaging of the sample. Fruitful discussions with Boris Luk'yanchuk and Reuben Bakker are greatly appreciated.

[1] J. N. Anker, W. P. Hall, O. Lyandres, N. C. Shah, J. Zhao, and R. P. Van Duyne, Biosensing with Plasmonic Nanosensors, Nat. Mater. 7, 442 (2008).

[2] A. V. Kabashin, P. Evans, S. Pastkovsky, W. Hendren, G. A. Wurtz, R. Atkinson, R. Pollard, V. A. Podolskiy, and A. V. Zayats, Plasmonic Nanorod Metamaterials for Biosensing, Nat. Mater. 8, 867 (2009).

[3] P. K. Jain, X. Huang, I. H. El-Sayed, and M. A. El-Sayed, Noble Metals on the Nanoscale: Optical and Photothermal Properties and Some Applications in Imaging, Sensing, Biology, and Medicine, Acc. Chem. Res. 41, 1578 (2008).

[4] Y. Choi, Y. Park, T. Kang, and L. P. Lee, Selective and Sensitive Detection of Metal Ions by Plasmonic Resonance Energy Transfer-Based Nanospectroscopy, Nat. Biotechnol. 4, 742 (2009).

[5] E. Jakeman and J. G. Rarity, The Use of Pair Production Processes to Reduce Quantum Noise in Transmission Measurements, Opt. Commun. 59, 219 (1986).

[6] M. M. Hayat, A. Joobeur, and B. E. A. Saleh, Reduction of Quantum Noise in Transmittance Estimation Using Photon-Correlated Beams, J. Opt. Soc. Am. A 16, 348 (1999).

[7] C. K. Hong, S. R. Friberg, and L. Mandel, Optical Communication Channel Based on Coincident Photon Pairs, Appl. Opt. 24, 3877 (1985).

[8] D. E. Chang, A. S. Sorensen, P. R. Hemmer, and M. D. Lukin, Quantum Optics with Surface Plasmons, Phys. Rev. Lett. 97, 053002 (2006).

[9] A. G. Curto, G. Volpe, T. H. Taminiau, M. P. Kreuzer, R. Quidant, and N. F. van Hulst, Unidirectional Emission of a Quantum Dot Coupled to a Nanoantenna, Science 329 , 930 (2010).

[10] S. Kumar, A. Huck, and U. L. Andersen, Efficient Coupling of a Single Diamond Color Center to Propagating Plasmonic Gap Modes, Nano Lett. 13, 1221 (2013). 
[11] R. Kolesov, B. Grotz, G. Balasubramanian, R. J. Stöhr, A. A. L. Nicolet, P. R. Hemmer, F. Jelezko, and J. Wrachtrup, Wave-Particle Duality of Single Surface Plasmon Polaritons, Nat. Phys. 5, 470 (2009).

[12] A. Huck, S. Kumar, A. Shakoor, and U. L. Andersen, Controlled Coupling of a Single Nitrogen-Vacancy Center to a Silver Nanowire, Phys. Rev. Lett. 106, 096801 (2011).

[13] J. T. Choy, B. J. M. Hausmann, T. M. Babinec, I. Bulu, M. Khan, P. Maletinsky, A. Yacoby, and M. Lončar, Enhanced Single-Photon Emission from a Diamond-Silver Aperture, Nat. Photonics 5, 738 (2011).

[14] R. M. Bakker, V. P. Drachev, Z. Liu, H.-K. Yuan, R. H. Pedersen, A. Boltasseva, J. Chen, J. Irudayaraj, A. V. Kildishev, and V. M. Shalaev, Nanoantenna Array-Induced Fluorescence Enhancement and Reduced Lifetimes, New J. Phys. 10, 125022 (2008).

[15] S. Schietinger, M. Barth, T. Aichele, and O. Benson, Plasmon-Enhanced Single Photon Emission from a Nanoassembled Metal-Diamond Hybrid Structure at Room Temperature, Nano Lett. 9, 1694 (2009).

[16] A. V. Akimov, A. Mukherjee, C. L. Yu, D. E. Chang, A. S. Zibrov, P. R. Hemmer, H. Park, and M. D. Lukin, Generation of Single Optical Plasmons in Metallic Nanowires Coupled to Quantum Dots, Nature (London) 450, 402 (2007).

[17] E. Alterwischer, M. P. van Exter, and J. P. Woerdman, Plasmon-Assisted Transmission of Entangled Photons, Nature (London) 418, 304 (2002).

[18] S. Fasel, F. Robin, E. Moreno, D. Erni, N. Gisin, and H. Zbinden, Energy-Time Entanglement Preservation in Plasmon-Assisted Light Transmission, Phys. Rev. Lett. 94, 110501 (2005).

[19] G. Fujii, T. Segawa, S. Mori, N. Namekata, D. Fukuda, and S. Inoue, Preservation of Photon Indistinguishability After Transmission through Surface-Plasmon-Polariton Waveguide, Opt. Lett. 37, 1535 (2012).

[20] A. Huck, S. Smolka, P. Lodahl, A. Sørensen, A. Boltasseva, J. Janousek, and U. Andersen, Demonstration of Quadrature-Squeezed Surface Plasmons in a Gold Waveguide, Phys. Rev. Lett. 102, 246802 (2009).

[21] B. J. Lawrie, P. G. Evans, and R. C. Pooser, Extraordinary Optical Transmission of Multimode Quantum Correlations via Localized Surface Plasmons, Phys. Rev. Lett. 110, 156802 (2013).

[22] R. W. Heeres, L. P. Kouwenhoven, and V. Zwiller, Quantum Interference in Plasmonic Circuits, Nat. Nanotechnol. 8, 719 (2013).

[23] B. Y. Zel'dovich and D. N. Klyshko, Statistics of Field in Parametric Luminescence, Sov. Phys. JETP Lett. 9, 40 (1969).

[24] D. C. Burnham and D. L. Weinberg, Observation of Simultaneity in Parametric Production of Optical Photon Pairs, Phys. Rev. Lett. 25, 84 (1970).

[25] M. B. Nasr, S. Carrasco, B. Saleh, A. Sergienko, M. Teich, J. Torres, L. Torner, D. Hum, and M. Fejer, Ultrabroadband Biphotons Generated via Chirped Quasi-Phase-Matched Optical Parametric Down-Conversion, Phys. Rev. Lett. 100, 183601 (2008).

[26] D. A. Kalashnikov, K. G. Katamadze, and S. P. Kulik, Controlling the Spectrum of a Two-Photon Field:
Inhomogeneous Broadening Due to a Temperature Gradient, JETP Lett. 89, 224 (2009).

[27] K. G. Katamadze and S. P. Kulik, Control of the Spectrum of the Biphoton Field, JETP 112, 20 (2011).

[28] M. Okano, R. Okamoto, A. Tanaka, S. Subashchandran, and S. Takeuchi, Generation of Broadband Spontaneous Parametric Fluorescence Using Multiple Bulk Nonlinear Crystals, Opt. Express 20, 13977 (2012).

[29] G. Scarcelli, A. Valencia, S. Gompers, and Y. Shih, Remote Spectral Measurement Using Entangled Photons, Appl. Phys. Lett. 83, 5560 (2003).

[30] A. Yabushita and T. Kobayashi, Spectroscopy by FrequencyEntangled Photon Pairs, Phys. Rev. A 69, 013806 (2004).

[31] A. A. Kalachev, D. A. Kalashnikov, A. A. Kalinkin, T. G. Mitrofanova, A. V. Shkalikov, and V. V. Samartsev, Biphoton Spectroscopy of YAG: $\mathrm{Er}^{3+}$ Crystal, Laser Phys. Lett. 4, 722 (2007).

[32] A. A. Kalachev, D. A. Kalashnikov, A. A. Kalinkin, T. G. Mitrofanova, A. V. Shkalikov, and V. V. Samartsev, Biphoton Spectroscopy in a Strongly Nondegenerate Regime of SPDC, Laser Phys. Lett. 5, 600 (2008).

[33] O. Slattery, L. Ma, P. Kuo, Y.-S. Kim, and X. Tang, Frequency Correlated Biphoton Spectroscopy Using Tunable Upconversion Detector, Laser Phys. Lett. 10, 075201 (2013).

[34] V. G. Kravetz, F. Schedin, and A. N. Grigorenko, Extremely Narrow Plasmon Resonances Based on Diffraction Coupling of Localized Plasmons in Arrays of Metallic Nanoparticles, Phys. Rev. Lett. 101, 087403 (2008).

[35] G. Vecchi, V. Giannini, and J. G. Rivas, Shaping the Fluorescent Emission by Lattice Resonances in Plasmonic Crystals of Nanoantennas, Phys. Rev. Lett. 102, 146807 (2009).

[36] G. Vecchi, V. Giannini, and J. G. Rivas, Surface Modes in Plasmonic Crystals Induced by Diffractive Coupling of Nanoantennas, Phys. Rev. B 80, 201401(R) (2009).

[37] A. I. Kuznetsov, A. B. Evlyukhin, M. R. Gonçalves, C. Reinhardt, A. Koroleva, M. L. Arnedillo, R. Kiyan, O. Marti, and B. N. Chichkov, Laser Fabrication of LargeScale Nanoparticle Arrays for Sensing Applications, ACS Nano 5, 4843 (2011).

[38] B. Luk'yanchuk, N. I. Zheludev, S. A. Maier, N. J. Halas, P. Nordlander, H. Giessen, and C. T. Chong, The Fano Resonance in Plasmonic Nanostructures and Metamaterials, Nat. Mater. 9, 707 (2010).

[39] A. E. Miroshnichenko, S. Flach, and Y. S. Kivshar, Fano Resonances in Nanoscale Structures, Rev. Mod. Phys. 82, 2257 (2010).

[40] Lord Reyleigh, On the Dynamical Theory of Gratings, Proc. R. Soc. A 79, 399 (1907).

[41] V. G. Kravets, F. Schedin, A. V. Kabashin, and A. N. Grigorenko, Sensitivity of Collective Plasmon Modes of Gold Nanoresonators to Local Environment, Opt. Lett. 35, 956 (2010).

[42] J. E. Mark, Polymer Data Handbook (Oxford University Press, New York, 1999).

[43] L. F. Hoyt, Determination of Refractive Index of Glycerols by the Immersion Refractometer, Oil Soap 10, 43 (1933).

[44] http://www.picoquant.com/products/category/photoncounting-detectors; http://www.picoquant.com/products/ category/tcspc-and-time-tagging-modules. 